\title{
A Survey of Face Recognition Methods
}

\author{
Li Xianwei \\ School of Information Engineering of \\ Suzhou University \\ Suzhou, Anhui Province,234000, China \\ lixianwei163@163.com
}

\author{
Zhang Haiyang \\ School of Earth Science Engineering of \\ Suzhou University \\ Suzhou, Anhui Province,234000, China \\ seazhang188@126.com
}

\begin{abstract}
Face recognition has received much attention because of its theoretical challenges as well as applications in user identification, surveillance and human-computer interaction. As a result, numerous methods have been developed for face recognition in the last few decades. Some universally used methods are introduced in this paper. On the same face recognition database, the recognition results are analyzed and compared.
\end{abstract}

Keywords-face recognition; machine learning; computer vision

\section{INTRODUCTION}

Face recognition has obtained an increasing amount of attention in machine learning and computer vision over the past few years. The reason is that face recognition technology can be applied in a wide range of fields, such as identity authentication, access control and so on [1].

Generally speaking, there are two categories of methods in face recognition [2]. One approach is based on facial feature. Firstly, the features such as eyes, nose and mouth first are located and then various feature extraction methods can be adopted to construct feature vectors of these facial features. Finally, traditional pattern recognition methods like a neural network can be used to recognize the feature vectors.

The other approach takes a holistic view of the recognition problem. It extracts the statistical characterization by the statistical method directly out of the entire training sample images instead of extracting the feature of the nose, mouth, or the eyes separately.

\section{REVIEW OF FACE RECOGNITION TECHNIQUES}

\section{A. Appearance-based face recognition}

Appearance-based face recognition can be divided into linear analysis methods such as PCA, ICA and LDA and non-linear analysis methods, such as KPCA.

Classical linear appearance-based analyses are PCA, ICA and LDA and each of them has its own basis vectors of a high dimensional face image space. The face vectors can be projected to the basis vectors by using those linear analysis methods.

Dimensionality of original input image space can be reduced through the projecting from a higher dimensional input image space to a lower dimensional space.

The matching score between the test face image and training images can be achieved by calculation the differences between their projection vectors. The higher the score, the more similar between these two face images.

1) Principal Component Analysis

Principal component analysis is used to calculate the vectors which best represent this small region of image space.It is also known as Karhunen-Loève expansion, eigenpicture, eigenvector, and principal Component [3-5]. The main idea of the principal component analysis is to find the vectors which best describe the distribution of face images within the entire image space. Its principle can be illustrated by Figure1. PCA is performed by projecting a new image into the subspace called face space spanned by the eigenfaces and then classifying the face by comparing its position in face space with the positions of known individuals.PCA aims to extract a subspace where the variance is maximized

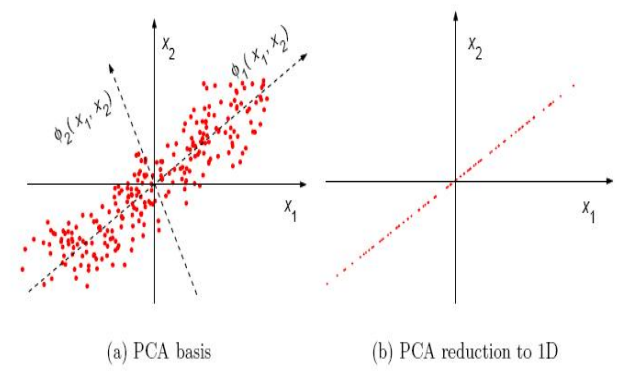

Figure1. The principle of PCA

2) Independent Component Analysis

PCA derives only the most expressive features which are unrelated to actual face recognition, and in order to improve performance additional discriminant analysis is needed. However, ICA provides a more powerful data representation than PCA as its aim is to provide an independent rather than uncorrelated image decomposition and representation. ICA is a generalization of PCA [6]. The principle of ICA can be described in Figure2.

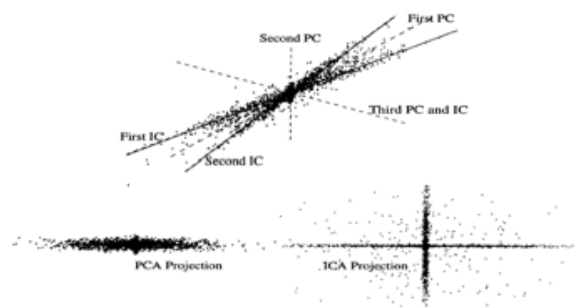

Figure2. The principle of ICA

3) Linear Discriminant Analysis 
Similar images projections are close together, different images projections locate far away when using PCA, but the projection from different classes of images are mixed together.

LDA is also called Fisher Discriminant Analysis. LDA is able to maximize the ratio of between-class distribution to that of within-class distribution [7, 8]. The principle of LDA can be illustrated in Figure3.

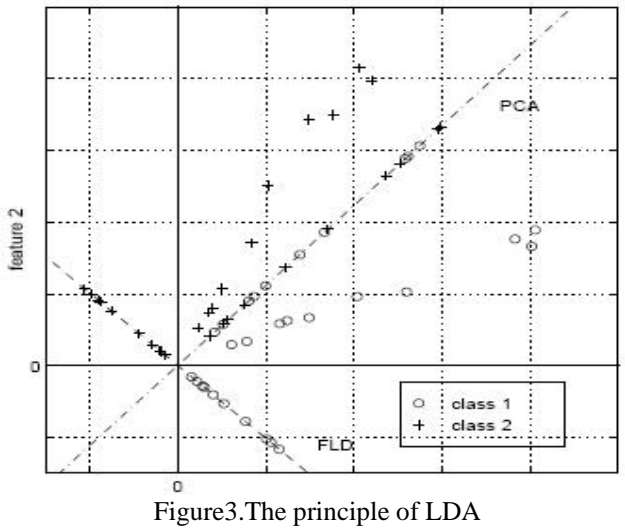

4) Nonlinear analysis

Linear discriminant methods are insensitive to the relationship among multiple pixels in the images. Some nonlinear relations may exist in a face image, especially under a complicated variation in viewpoint, illumination and facial expression which is highly nonlinear.

To extract nonlinear features of images, linear analysis method was extended to nonlinear analysis such as Kernel PCA, Kernel ICA and Kernel FLD etc [9, 10].

By using nonlinear analysis approaches the original input image space is projected nonlinearly onto a high dimensional feature space. In this high dimensional space, the distribution of image vectors could be simplified to linear pattern. Comparison of PCA and KPCA can be demonstrated in Figure3.

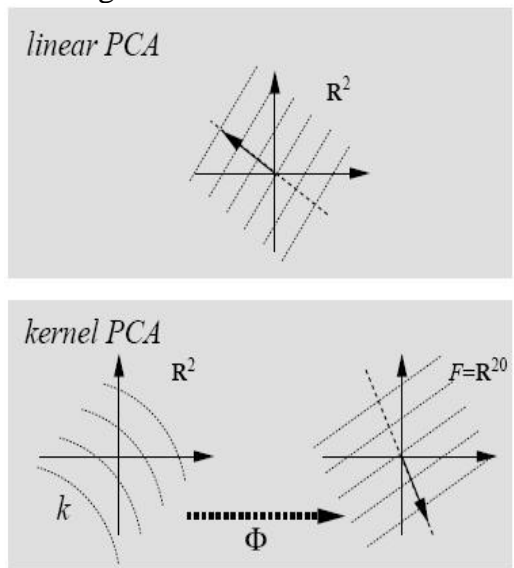

Figure3. Comparison of PCA and KPCA

\section{B. Neural Networks}

The attractiveness of using neural networks could be due to its non linearity in the network. Hence, the feature extraction step may be more efficient than the linear
Karhunen-Loève

methods. One of the first artificial neural networks (ANN) techniques used for face recognition is a single layer adaptive network called WISARD which contains a separate network for each stored individual [11]. The way in constructing a neural network structure is crucial for successful recognition. It is very much dependent on the intended application. For face detection, multilayer perception [12] and convolutional neural network [13] have been applied. Reference [13] proposed a hybrid neural network which combines local image sampling, a self-organizing map (SOM) neural network, and a convolutional neural network. The SOM provides a quantization of the image samples into a topological space where inputs that are nearby in the original space are also nearby in the output space, thereby providing dimension reduction and invariance to minor changes in the image sample. A simple neural network is described in Figure4.

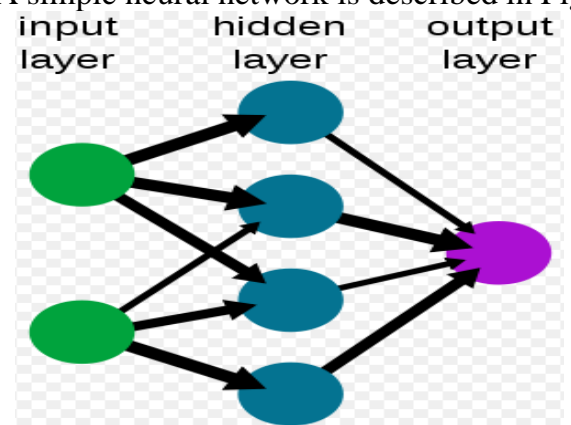

Figure4.A simple neural network

\section{Hidden Markov Models (HMMs)}

Stochastic modeling of nonstationary vector time series based on (HMM) has been very successful for speech applications. Reference [14] applied this method to human face recognition. Faces were intuitively divided into regions such as the eyes, nose, mouth, etc., which can be associated with the states of a hidden Markov model. Since HMMs require a one-dimensional observation sequence and images are two-dimensional, the images should be converted into either 1D temporal sequences or 1D spatial sequences.

\section{Geometrical Feature Matching}

Geometrical feature matching techniques are based on the computation of a set of geometrical features from the picture of a face. The fact that face recognition is possible even at coarse resolution as low as $8 \times 6$ pixels [15] when the single facial features are hardly revealed in detail implies that the overall geometrical configuration of the face features is sufficient for recognition. The overall configuration can be described by a vector representing the position and size of the main facial features, such as eyes and eyebrows, nose, mouth, and the shape of face outline.

\section{E. Support Vector Machine (SVM)}

Applying SVM to computer vision problem has been proposed in recent years. Osuna et al [16] train a SVM for face detection, where the discrimination is between two classes: face and non-face, each with thousands of examples. 
For a two-class classification problem, the goal is to separate the two classes by a function which is induced from available examples.SVM is a learning technique that is considered an effective method for general purpose pattern recognition because of its high generalization performance without the need to add other knowledge [17]. Intuitively, given a set of points belonging to two classes, a SVM finds the hyperplane that separates the largest possible fraction of points of the same class on the same side, while maximizing the distance from either class to the hyperplane. According to [17], this hyperplane is called Optimal Separating Hyperplane (OSH) which minimizes the risk of misclassifying not only the examples in the training set but also the unseen example of the test set.

\section{CONCLUSIONS}

Some classical face recognition methods are presented in this paper. On the same face database, the methods of SVM and HMM can get better face recognition results, but the algorithms are more complex.

\section{REFERENCES}

[1]R. Chellappa, C.L. Wilson, S. Sirohey, "Human and Machine Recognition of Faces: A Survey,” Proceedings of the IEEE, Vol. 83, No.5, May.1995, pp. 705-740.

[2]S. Ranganath and K. Arun, "Face Recognition Using Transform Features and Neural Network,” Pattern Recognition, Vol..30, Oct.1997,pp. 1615-1622.

[3] Matthew A. Turk, Alex P. Pentland, "Face Recognition Using Eigenfaces," Proc. IEEE Conference on Computer Vision and Pattern Recognition: 586-591. 1991.

[4] M. Kirby and L. Sirovich, "Application of the Karhunen- Loève procedure for the characterisation of human faces," IEEE Trans.

Pattern Analysis and Machine Intelligence, vol. 12, pp. 831-835, Dec.1990.

[5]L. Sirovich and M. Kirby, "Low-Dimensional procedure for the characterization of human faces,” J. Optical Soc. of Am., vol. 4, pp.519-524, 1987.

[6] C. Liu, H. Wechsler, Comparative Assessment of Independent Component Analysis (ICA) for Face Recognition, Proc. of the Second International Conference on Audio- and Video-based Biometric Person Authentication, AVBPA'99, 22-24 March 1999, Washington D.C., USA, pp. 211-216

[7]A.M. Martinez, A.C. Kak, PCA versus LDA, IEEE Trans. on Pattern Analysis and Machine Intelligence, Vol. 23, No. 2, 2001, pp. 228-233

[8]J. Lu, K.N. Plataniotis, A.N. Venetsanopoulos, Face Recognition Using LDA-Based Algorithms, IEEE Trans. on Neural Networks, Vol. 14, No. 1, January 2003, pp. 195-200

[9]M.-H. Yang, Kernel Eigenfaces vs. Kernel Fisherfaces: Face Recognition Using Kernel Methods, Proc. of the Fifth IEEE International Conference on Automatic Face and Gesture Recognition, 20-21 May 2002, Washington D.C., USA, pp. 215-220

[10]H. Yang, Face Recognition Using Kernel Methods, Advances in Neural Information Processing Systems, T. Diederich, S. Becker, Z. Ghahramani, Eds., 2002, vol. 14, 8 pages

[11] T.J. Stonham, "Practical face recognition and verification with WISARD,” Aspects of Face Processing, pp. 426-441, 1984.

[12] K.K. Sung and T. Poggio, "Learning human face detection in cluttered scenes," Computer Analysis of Image and patterns, pp. 432-439, 1995.

[13] S. Lawrence, C.L. Giles, A.C. Tsoi, and A.D. Back, "Face recognition:A convolutional neural-network approach,” IEEE Trans. Neural Networks, vol. 8, pp. 98-113, 1997.

[14] F. Samaria and A.C. Harter, "Parameterisation of a stochastic model for human face identification,” Proc. Second IEEE Workshop Applications of Computer Vision, 1994.

[15] S. Tamura, H. Kawa, and H. Mitsumoto, "Male/Female identification from 8_6 very low resolution face images by neural network," Pattern Recognition, vol. 29, pp. 331-335, 1996.

[16] E. Osuna, R. Freund, and F. Girosi, "Training support vector machines: An application to face detection," Proc. Computer Vision and Pattern Recognition, pp.130-136, San Juan, 1997.

[17]V.N. Vapnik, “The nature of statistical learning theory," New York:Springverlag, 1995. 